\title{
Ventricular asynchrony: A shift to the right?
}

\author{
Andrew Van Tosh, ${ }^{\mathrm{a}, \mathrm{b}}$ and Kenneth J. Nichols ${ }^{\mathrm{c}, \mathrm{d}}$ \\ a Research Department, St. Francis Hospital-The Heart Center, Roslyn, NY \\ ${ }^{b}$ St. Francis Hospital, Roslyn, NY \\ ${ }^{c}$ Division of Nuclear Medicine and Molecular Imaging, North Shore - Long Island Jewish Health \\ System, Manhasset, NY \\ d Division of Nuclear Medicine and Molecular Imaging, North Shore - Long Island Jewish Health \\ System, New Hyde Park, NY
}

Received Dec 11, 2015; accepted Dec 11, 2015

doi: $10.1007 / \mathrm{s} 12350-015-0383-1$

\section{See related article, pp. 69-78}

Evaluation of ventricular function has been a focus of nuclear medicine since 1971, when Zaret et al demonstrated that abnormalities of left ventricular (LV) wall motion and ejection fraction (EF) could be determined by injection of Tc-99m human serum albumen and acquiring images at end-diastole and end-systole. ${ }^{1}$ Multi-gated equilibrium radionuclide ventriculography (RNV) and first-pass imaging further refined characterization of LV asynergy and performance. ${ }^{2,3}$ Clinical trials soon showed that noninvasively determined LVEF was a strong predictor of survival in a broad range of heart diseases. ${ }^{4,5}$ Further work demonstrated that synchronicity of LV contraction could be derived from RNV by Fourier analysis of pixel by pixel labeled RBC time-activity curves, assigning a phase to each pixel (percent of the R-R interval from $0^{\circ}-360^{\circ}$ ) to identify time of maximum contraction. ${ }^{6}$ Synchronicity has important effects on LV performance: patients with inter- or intra-ventricular asynchrony have lower LVEF relative to normal control subjects. ${ }^{7}$ Mechanisms for reduced ventricular performance resulting from dyssynchrony remain unclear, but Sweeney et al suggested early-contracting segments stretch and deform later-contracting ones, and vice versa, expending energy in the process, resulting in lower rate of pressure rise, lower developed pressure, prolonged ejection, and reduced $E F{ }^{8}$ The most ubiquitous methods to measure

Reprint requests: Andrew Van Tosh, Research Department, St. Francis Hospital-The Heart Center, Roslyn, NY; andrew.vantosh@chsli.org J Nucl Cardiol 2017:24;79-82.

1071-3581/\$34.00

Copyright (C) 2016 American Society of Nuclear Cardiology. asynchrony are by tissue doppler echocardiographic techniques, which use low frequency signals to measure myocardial wall deformation, and calculate strain, strain rate, delay in contraction of opposing LV walls (four basal LV segments on 4-chamber view), and dispersion of time to peak systolic contraction. ${ }^{9}$

Cardiac resynchronization therapies (CRT) were designed to improve LV synchronicity through optimally timed pacing (as determined by echo) of right ventricle (RV) and LV lateral walls. CRT promotes reverse remodeling, with improved EF and survival in heart failure patients. ${ }^{10}$ However, clinical response to CRT (defined hemodynamically or as decrease in NYHA CHF class) occurs in only two-thirds of patients, prompting efforts to identify variables that would prospectively predict a positive CRT response. Multicenter studies ("Prospect" and "Rethinq" trials) demonstrated large variability in performance of echo parameters, and their failure to predict CRT response, ${ }^{11,12}$ which fostered the development of nuclear cardiology methods for dyssynchrony assessment.

One nuclear approach to quantifying asynchrony used gated myocardial perfusion imaging (GMPI) SPECT data. ${ }^{13}$ Because of partial volume effects, myocardial wall thickening varies linearly with systolic counts, ${ }^{14}$ so that onset of mechanical contraction (OMC) in each LV myocardial pixel was defined as increase of systolic counts from baseline. Fourier analysis generated curves of OMC phase per pixel, plotted as frequency histograms, the standard deviation (SD) and bandwidth (BW) of which were quantified. A slightly different approach that imposes constant myocardial mass constraints was developed for Quantitative Gated SPECT (QGS) software, ${ }^{15}$ the BW and SD measurements of which successfully separated normal patients from those with LBBB. ${ }^{16}$ Correlations between phase parameters from GMPI SPECT and tissue Doppler were modest, 
partly because GMPI phase is a global measurement, while TDE represents single points and parameters. ${ }^{17}$ GMPI phase SD and BW have the advantage of being highly reproducible and repeatable. ${ }^{18,19}$ Single center studies indicated GMPI phase parameters can help identify patients likely to respond to CRT, with GMPI SPECT providing additional information on location and extent of myocardial infarction/scarring, which together with location of the LV pacing lead predicts CRT response with positive predictive value of $96 \%{ }^{20}$

Evaluation of RV function has been more difficult than the LV because of RV anatomy. The RV is pyramidal in shape, with an inlet, a main body, and outflow tract, so that borders are difficult to outline. Also, RV wall thickness typically is a third of that of the LV, exacerbating nuclear imaging difficulties. Nonetheless, quantifying RV systolic function is important, as it predicts outcome in valvular and ischemic heart disease. ${ }^{21}$ Using first-pass RNV, Brent et al showed that patients with pulmonary hypertension had diminished RVEF both at rest and in response to exercise. ${ }^{22}$ SPECT RNV RVEF and volumes are strongly correlated with those determined by MR in patients with pulmonary hypertension and Tetralogy of Fallot. ${ }^{23}$

2D echocardiography is frequently used to evaluate $\mathrm{RV}$ function, via change in sectional RV area from the four chamber view, and by measuring velocity of the tricuspid annulus. However, RV 2D echo measurements correlate only modestly with other methods. ${ }^{24} 3 \mathrm{D}$ echocardiography has been more effective, and has been successfully used to establish normal values for RVEF and volumes. ${ }^{25}$ In patients with varying degrees of pulmonary hypertension, RV dyssynchrony parameters are strong and independent predictors of outcomes. Tissue Doppler imaging has been used to evaluate RV asynchrony, with speckle tracking used to measure time to peak systolic strain for the RV free wall, or four segments in the mid to basal RV on four chamber view. ${ }^{26}$

In patients with pulmonary hypertension, scintigraphy has shown a D-shape septum and increased RV uptake of perfusion tracers, which correlates with pulmonary artery pressure, ${ }^{27}$ and that resting $\mathrm{RV}$ myocardial blood flow by N-13 PET was half that of the $\mathrm{LV}^{28}$ Moreover, RV glucose uptake was $70 \%$ of the $\mathrm{LV}$, making $\mathrm{N}-13$ an attractive isotope for $\mathrm{RV}$ functional measurements.

In this issue, Wang et al have made an important contribution to evaluation of RV function, ${ }^{29}$ constituting the first study analyzing RV asynchrony in pulmonary hypertension patients using F-18-FDG PET. This study evaluated whether FDG PET RV asynchrony parameters correlate with those derived from speckle tracking echocardiography and with clinical and hemodynamic predictors of outcome.
Methodologically, the authors repositioned the Emory Toolbox Synchtool's circular region of interest to encompass the septum, RV free wall, and diaphragmatic wall. A phase polar coordinate map was constructed, in which the septum was represented on the right-most area, and the RV free wall and diaphragmatic walls in anterior and inferior locations. Phase SD and BW, and the time delay from onset of RV free wall contraction to that of the septum, were calculated. Echo speckle tracking was then used to determine the same time delay, and these two methods were then compared.

There were several important findings from their data. First, there was excellent correspondence between the order of onset of contraction in the septum and free wall determined by PET phase measurements versus speckle tracking. Also, there was significant correlation between phase delay on PET and speckle tracking. This finding provides independent support for the validity of the RV nuclear phase measurements. There were also significant correlations between RV phase SD and BW and RVEF, C.I., BNP, and time on the 6 min walk test. Interestingly, there was no correlation between dyssynchrony parameters and RV hemodynamics, which was ascribed to heterogeneity of the population.

This investigation represents a logical next step in evaluating asynchrony by nuclear techniques by exploring the feasibility of extending asynchrony analysis to the RV by FDG PET. While this study provides convincing echocardiographic data that supports the approach, more work needs to be done before these methods can be considered well-validated. Reproducibility of these RV techniques needs to be established, including inter- and intra-observer reproducibility, as has been done for LV parameters. ${ }^{18,19}$ The longer half-life of F-18, relative to other PET tracers, is well suited to performing two acquisitions from a single injection. Also, correlations in this study between the time differential of RV septal to free wall contraction for PET versus echo is significant, but moderate at best. It is acknowledged that this may be due in part to PET measuring the phase onset of mechanical contraction, while echo speckle tracking measures time to peak strain, somewhat different parameters. In a larger sense, the free wall to septal delay measurement is a single plane radial strain parameter, while nuclear dyssynchrony measurements reflect global dispersion of contraction phase. The next step in the validation process would be to compare asynchrony by tomographic FDG PET against 3D echo asynchrony parameters.

A further concern is that the Synchtool uses a circular region of interest developed to define the short axis LV. However, the RV is not circular, which may introduce distortion and inaccuracy. The septal phase obtained from the RV should be compared to that of the 
LV, as these should be identical; such an analysis would serve as processing quality control.

$\mathrm{RV}$ asynchrony offers important prognostic information in patients with pulmonary hypertension, and the current study indicates that FDG PET RV asynchrony correlates with RVEF and other predictors of prognosis in pulmonary hypertension. Future studies are needed to determine whether FDG PET RV asynchrony offers incremental prognostic information in this group. LV asynchrony is recognized as an important component of LV dysfunction, while the contribution of RV asynchrony to left-sided heart failure has been less explored. The current study has focused on RV asynchrony in pulmonary hypertension, because RV hypertrophy results in count rates approaching that of the LV, making asynchrony analysis more feasible. However, patients with left-sided CHF may also have pulmonary hypertension, RV hypertrophy, and increased RV:LV count ratios. In addition, such patients may often undergo PET FDG studies for viability determination, or scar quantitation, before CRT implementation. Those clinical circumstances may offer an ideal opportunity to assess whether RV asynchrony can be quantified by PET FDG in a general heart failure population, and determine if these measurements have a role in the evaluation for CRT.

\section{References}

1. Zaret BL, Strauss HW, Hurley PJ, Natarajan TK, Pitt B. A noninvasive scintiphotographic method for detecting regional ventricular dysfunction in man. N Engl J Med 1971;284:1165-71.

2. Bacharach SL, Green MV, Borer JS, Ostrow HG, Redwood DR, Johnston GS. ECG-gated scintillation probe measurement of left ventricular function. J Nucl Med 1977;18:1176-81.

3. Walton S, Ell PJ, Jarritt PH, Swanton RH. Phase analysis of the first pass radionuclide angiocardiogram. Br Heart J 1982;48:441-8.

4. Supino PG, Wallis JB, Chlouverakis G, Borer JS. Risk stratification in the elderly patient after coronary artery bypass grafting: The prognostic value of radionuclide cineangiography. J Nucl Cardiol 1994;1:159-70.

5. Supino PG, Borer JS, Herrold EM, Hochreiter C. Prognostication in 3-vessel coronary artery disease based on left ventricular ejection fraction during exercise: Influence of coronary artery bypass grafting. Circulation. 1999;100:924-32.

6. O'Connell JW, Schreck C, Moles M, Badwar N, DeMarco T, Olgin J, et al. A unique method by which to quantitate synchrony with equilibrium radionuclide angiography. J Nucl Cardiol 2005; 12:441-50.

7. Grines CL, Bashore TM, Boudoulas H, Olson S, Shafer P, Wooley CF. Functional abnormalities in isolated left bundle branch block The effect of interventricular asynchrony. Circulation 1989;79:845-53.

8. Sweeney MO, Hellkamp AS. Heart failure during cardiac pacing. Circulation 2006;113:2082-8.

9. Yu CM, Chau E, Sanderson JE, Fan K, Tang MO, Fung WH, et al. Tissue Doppler echocardiographic evidence of reverse remodeling and improved synchronicity by simultaneously delaying regional contraction after biventricular pacing therapy in heart failure. Circulation 2002;105:438-45.

10. Cleland JG, Daubert JC, Erdmann E, Freemantle N, Gras D, Kappenberger L, et al. The effect of cardiac resynchronization on morbidity and mortality in heart failure. $\mathrm{N}$ Engl $\mathrm{J}$ Med 2005;352:1539-49.

11. Chung ES, Leon AR, Tavazzi L, Sun JP, Nihoyannopoulos P, Merlino J, et al. Results of the predictors of response to CRT (PROSPECT) trial. Circulation 2008;117:2608-16.

12. Beshai JF, Grimm RA, Nagueh SF, Baker JH, Beau SL, Greenberg SM, et al. Cardiac-resynchronization therapy in heart failure with narrow QRS complexes. N Engl J Med 2007;357:2461-71.

13. Chen J, Garcia EV, Folks RD, Cooke CD, Faber TL, Tauxe EL, et al. Onset of left ventricular mechanical contraction as determined by phase analysis of ECG-gated myocardial perfusion SPECT imaging: Development of a diagnostic tool for assessment of cardiac mechanical dyssynchrony. J Nucl Cardiol 2005;12:68795.

14. Galt JR, Garcia EV, Robbins WL. Effects of myocardial wall thickness on SPECT quantification. IEEE Trans Med Imaging 1990;9:144-50.

15. Boogers MM, Van Kriekinge SD, Henneman MM, Ypenburg C, Van Bommel RJ, Boersma E, et al. Quantitative gated SPECTderived phase analysis on gated myocardial perfusion SPECT detects left ventricular dyssynchrony and predicts response to cardiac resynchronization therapy. J Nucl Med 2009;50:718-25.

16. Van Kriekinge SD, Nishina H, Ohba M, Berman DS, Germano G. Automatic global and regional phase analysis from gated myocardial perfusion SPECT imaging: Application to the characterization of ventricular contraction in patients with left bundle branch block. J Nucl Med 2008;49:1790-7.

17. Daya HA, Malhotra S, Soman P. Radionuclide assessment of left ventricular dyssynchrony. Cardiol Clin 2016;34:101-18.

18. Trimble MA, Velazquez EJ, Adams GL, Honeycutt EF, Pagnanelli RA, Barnhart HX, et al. Repeatability and reproducibility of phase analysis of gated single-photon emission computed tomography myocardial perfusion imaging used to quantify cardiac dyssynchrony. Nucl Med Commun 2008;29:374-81.

19. Lin X, Xu H, Zhao X, Folks RD, Garcia EV, Soman P, et al. Repeatability of left ventricular dyssynchrony and function parameters in serial gated myocardial perfusion SPECT studies. J Nucl Cadiol 2010;17:811-6.

20. Friehling M, Chen J, Saba S, Bazaz R, Schwartzman D, Adelstein $\mathrm{EC}$, et al. A prospective pilot study to evaluate the relationship between acute change in left ventricular synchrony after cardiac resynchronization therapy and patient outcome using a single-injection gated SPECT protocol. Circ Cardiovasc Imaging 2011;4:532-9.

21. Hochreiter C, Niles N, Devereux RB, Kligfield P, Borer JS. Mitral regurgitation: Relationship of non-invasive descriptors of right and left ventricular performance to clinical and hemodynamic findings and to prognosis in medically and surgically treated patients. Circulation 1986;73:900-12.

22. Brent BN, Mahler D, Matthay RA, Berger HJ, Zaret BL. Noninvasive diagnosis of pulmonary arterial hypertension in chronic obstructive pulmonary disease: Right ventricular ejection fraction at rest. Am J Cardiol 1984;53:1349-53.

23. Nichols K, Saouaf R, Ababneh AA, Barst RJ, Rosenbaum MS, Groch MW, et al. Validation of SPECT equilibrium radionuclide angiographic right ventricular parameters by cardiac magnetic resonance imaging. J Nucl Cardiol 2002;9:153-60.

24. Reichek N. Right ventricular strain in pulmonary hypertension: Flavor du jour or enduring prognostic index? Circ Cardiovasc Imaging 2013;6:609-11. 
25. Maffessanti F, Muraru D, Esposito R, Gripari P, Ermacora D, Santoro C, et al. Age-, body size-, and sex-specific reference values for right ventricular volumes and ejection fraction by threedimensional echocardiography: A multicenter echocardiographic study in 507 healthy volunteers. Circ Cardiovasc Imaging 2013;6:700-10.

26. Smith BC, Dobson G, Dawson D, Charalampopoulos A, Grapsa J, Nihoyannopoulos P. Three-dimensional speckle tracking of the right ventricle: Toward optimal quantification of right ventricular dysfunction in pulmonary hypertension. J Am Coll Cardiol 2014;64:41-51.

27. Movahed MR, Hepner A, Lizotte P, Milne N. Flattening of the interventricular septum (D-shaped left ventricle) in addition to high right ventricular tracer uptake and increased right ventricular volume found on gated SPECT studies strongly correlates with right ventricular overload. J Nucl Cardiol 2005;12:428-34.

28. Bokhari S, Raina A, Rosenweig EB, Schulze PC, Bokhari J, Einstein AJ, et al. PET imaging may provide a novel biomarker and understanding of right ventricular dysfunction in patients with idiopathic pulmonary arterial hypertension. Circ Cardiovasc Imaging 2011;4:641-7.

29. Wang L, Zhou W, Liang Y, Yang Y, Garcia EV, Chen J, et al. Right ventricular dyssynchrony in pulmonary hypertension: Phase analysis using FDG-PET imaging. J Nucl Cardiol 2015. doi: 10.1007/s12350-015-0341-y. 